\title{
Capacidad de TECNOLOGÍAs DE INFORMACIÓN Y DESEMPEÑO ORGANIZACIONAL: EFECTO MEDIADOR DE LA CAPACIDAD DE ABSORCIÓN*
}

\author{
Santiago Guisao Paniagua ** \\ Luis David Rincón García

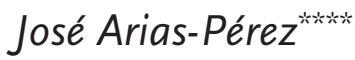

\footnotetext{
* doi: 10.11144/Javeriana.cao30-55.ctido. Este artículo es producto del trabajo de grado “Efecto mediador de la capacidad de absorción en la relación entre capacidad de tecnologías de información y desempeño organizacional financiero y no financiero", para optar al título de magíster en Administración, el cual contó con financiación del Departamento de Ciencias Administrativas de la Universidad de Antioquia, y se realizó entre febrero de 2015 hasta junio de 2016. El artículo se recibió el 03/08/2017 y se aprobó el 25/11/2017. Sugerencia de citación: Guisao P., S., Rincón G., L. y Arias-Pérez, J. (2017). Capacidad de tecnologías de información y desempeño organizacional: efecto mediador de la capacidad de absorción. Cuadernos de Administración, 30(55), 37-65. http:// dx.doi.org/10.11144/Javeriana.cao30-55.ctido.

** Magíster en Administración de la Universidad de Antioquia, Medellín, Colombia, 2016. Coordinador Producción Anestésicos, New Stetic S.A, Medellín, Colombia.

Correo electrónico: santiagog28@gmail.com

*** Magíster en Administración, Universidad de Antioquia, 2016. Director Administrativo y contable, Penta Alliance, Medellín, Colombia.

Correo electrónico: kamusd@gmail.com

**** Doctorando en Dirección de Empresas de la Universidad de Valencia, Valencia, España, 2018. Profesor del Departamento de Ciencias Administrativas de la Universidad de Antioquia, Medellín, Colombia.

Correo electrónico:jenrique.arias@udea.edu.co
} 


\section{Capacidad de tecnologías de información \\ y desempeño \\ organizacional: efecto mediador de la capacidad de absorción}

\section{RESUMEN}

El objetivo de este artículo es analizar el efecto mediador de la Capacidad de Absorción ( $C A B$ ) en la relación entre las Capacidades de Tecnologías de Información (CTI) y el Desempeño Organizacional, particularmente el desempeño financiero (DF) y el desempeño no financiero (DnF). El modelo de hipótesis se contrastó en una muestra de empresas manufactureras y de servicios, para ello, se utilizaron ecuaciones estructurales por el método de mínimos cuadrados parciales. Se encontró que existe una mediación total de CAB en la relación entre CTI y DF, y parcial entre CTI y DnF. En conclusión, para que las CTI logren incidir sobre los resultados empresariales, es necesaria su articulación con habilidades organizacionales blandas como la CAB.

Palabras clave: Tecnologías de información, economía emergente, gestión de conocimiento, estrategia de negocios.

Clasificación JEL: 014, 032, M15

\section{Capacity for information technologies and organizational performance: mediating effect of the absorption capacity}

\section{ABSTRACT}

The aim of this paper is to analyze the mediating effect of Absorptive Capacity (AC) in the relationship between Information Technology Capabilities (ITC) and Organizational Performance, particularly financial performance (FP) and non-financial performance (NFP). The hypothesis model was tested on a sample of manufacturing and service companies, for which a structural equation by the least squares method was used. As for the results it was found that there is a total mediation of $A C$ in the relationship between ITC and FP, and a partial one between ITC and NFP. In conclusion, to achieve the ITC impact on business results, its articulation is needed with soft organizational skills such as AC.

Keywords: Information technologies, emerging economy, knowledge management, business strategy.

JEL Classification: 014, 032, M15

\section{Capacidade de tecnologias da informação e desempenho organizacional: efeito mediador da capacidade de absorção}

\section{Resumo}

O objetivo deste artigo é analisar o efeito mediador da Capacidade de Absorção (CAB) na relação entre as Capacidades de Tecnologias da Informação (CTI) e o Desempenho Organizacional, particularmente o Desempenho Financeiro (DF) e o Desempenho não Financeiro (DnF). O modelo de hipótese contrastou-se numa amostra de empresas manufatureiras e de serviços; para isso, foram utilizadas equações estruturais pelo método de mínimos quadrados parciais. Quanto aos resultados, constatou-se que existe uma mediação total de $C A B$ na relação entre CTI e DF, e parcial entre CTI e DnF. Em conclusão, para que as CTI possam incidir sobre os resultados empresariais, é necessária sua articulação com habilidades organizacionais flexíveis como a CAB.

Palavras-chave: tecnologias da informação, economia emergente, gestão de conhecimento, estratégia de negócios. Classificação JEL: 014, 032, M15 


\section{Introducción}

En el entorno globalizado las organizaciones deben fortalecer las estrategias de implementación y desarrollo de las tecnologías de información (TI) con el fin de optimizar y robustecer cada uno de los procesos y generar valor, que se traduce en rendimiento y competitividad (WEF, 2015). Por ende, en la literatura ha surgido un interés por explorar el impacto de la relación entre las capacidades de tecnologías de información (CTI) y el desempeño organizacional (D0) (Bharadwaj, 2000; Santhanam y Hartono, 2003; Liu et al., 2013), entendidas las primeras como la capacidad de una empresa para adquirir, combinar, reconfigurar, movilizar y desplegar constantemente los recursos de TI en respuesta al entorno dinámico para mejorar los procesos de trabajo y estrategias de negocio, y así generar valor agregado y lograr una ventaja competitiva difícil de imitar o sustituir. A su vez, el D0 se entiende como un constructo multidimensional compuesto por un conjunto de resultados objetivos y subjetivos que son medidos por indicadores financieros y no financieros del negocio (Nakata et al., 2008; Liu et al., 2013).

Los estudios sobre la relación entre CTI y D0 han logrado demostrar que esta habilidad organizacional puede proporcionar mayores beneficios económicos, reducción de costos de comercialización (Chae et al., 2014), y por ende un rendimiento financiero superior (Bharadwaj, 2000). También genera activos intangibles tales como la diferenciación de productos y servicios (Tan y Teo, 2000), las patentes (Fahy y Hooley, 2002), el aumento de la lealtad del cliente, know-how, la reputación, el mejoramiento del servicio al cliente y de la calidad del producto y el aumento de la capacidad de respuesta del mercado (Brynjolfsson y Hitt, 1997).

De otro lado, estudios recientes presentan resultados contradictorios que indican la inexistencia de la relación entre CTI y D0, dado que a medida que aumenta la accesibilidad y disponibilidad de las TI y su costo disminuye, se convierten en un estándar de la industria, sin posibilidades de proporcionar diferenciación (Chae et al., 2014). En detalle, hay estudios que han demostrado que a mayor inversión de TI, el DO es el mismo y los índices de satisfacción de los clientes y de rentabilidad continúan estáticos, mostrando una incidencia estadísticamente no significativa de las CTI sobre el DO (Brynjolfsson y Hitt, 2000; Shin, 2001; Dedricky Kraemer, 2003; Francalanci y Morabito, 2008; Liu et al., 2013). Sin embargo, estos estudios se han desarrollado en países con un alto grado de incorporación de TIC en los negocios en comparación con países emergentes, dado que en el caso de los primeros en años recientes el índice de disponibilidad de TI ha estado por encima de 5,0 mientras que en los segundos se ha mantenido entre 3,5 y 3,8 (WEF, 
2015). Por esto, sería pertinente contar con estudios que aporten evidencia empírica concluyente en torno a esta relación en el contexto de países emergentes, en donde las TI sí podrían seguir siendo una fuente de ventaja competitiva (Liu et al., 2008).

De otra parte, ha surgido otra línea de investigación que explora la existencia de variables mediadoras en la relación entre CTI y D0, entre ellas, el aprendizaje organizacional (Tippins y Sohi, 2003; Yu y Xin-quan, 2011), la gestión del conocimiento (Tanriverdi, 2005) y la ambidestreza (Datta, 2012). Uno de esos constructos que se ha venido planteando como posible mediador es la Capacidad de Absorción (CAB), entendida como la habilidad organizacional para identificar, adquirir y capturar conocimiento valioso de fuentes externas, asimilarlo, transformarlo y explotarlo (Cohen y Levinthal, 1990). En ese sentido, existen estudios que se han aproximado tangencialmente al análisis de la mediación de la CAB, pero con énfasis en los aspectos duros de la CTI, relacionados con la integración de sistemas de información (Francalanci y Morabito, 2008; Liu et al., 2013). Por ende, la mediación de la CAB se ha planteado dando énfasis a la dimensión de infraestructura de TI, pero pasando por alto otros aspectos de CTI como la proactividad y la capacidad de generar valor a partir de TI (Lu y Ramamurthy, 2011). De otro lado, estos estudios se han concentrado en determinar el impacto sobre el desempeño financiero (DF), sin considerar de manera específica aspectos relacionados con el desempeño no financiero (DnF), como la satisfacción del cliente, el valor de las marcas, entre otros.

Por lo anterior, el objetivo del presente artículo es analizar la mediación de la CAB, en la relación entre CTI y D0, pero en un sentido amplio en el que las CTI se conciban con sus tres dimensiones: capacidad de infraestructura de TI, capacidad de TI para la expansión del negocio y capacidad de proactividad de TI (Lu y Ramamurthy, 2011). Igualmente, por el lado del DO se consideran aspectos financieros, pero también de manera específica los no financieros como la satisfacción del cliente, la imagen corporativa, el valor de las marcas y la productividad de los empleados (Lee et al., 2011; Camisón y Villar-López, 2014).

\section{Marco teórico}

\subsection{Capacidades de la tecnología de la información}

Las capacidades de la tecnología de la información (CTI) aluden a las habilidades de una empresa para integrar e implementar sus recursos informáticos y así cumplir con las necesidades y objetivos del negocio. En este sentido, las CTI se componen de tres 
dimensiones: la capacidad de la infraestructura de TI (la base tecnológica), la capacidad de TI de expansión del negocio y la postura proactiva en TI (Lu y Ramamurthy, 2011).

La capacidad de la infraestructura de TI se define como la habilidad organizacional para implementar plataformas donde se almacena y procesa toda la información, permitiendo que sea compartida de una manera más precisa y oportuna. En cambio, la capacidad de TI de expansión del negocio, es entendida como la habilidad organizacional para gestionar, visualizar y explotar los recursos de TI usándolos como apoyo para lograr con facilidad el cumplimiento de los objetivos de la compañía. Por su parte, la postura proactiva en TI consiste en la habilidad de buscar activamente las innovaciones en TI y adaptarlas de manera eficiente al ambiente y entorno organizacional, con el fin de generar oportunidades de negocio a partir de las herramientas de TI existentes (Lu y Ramamurthy, 2011).

\subsection{Desempeño financiero y no financiero}

El desempeño organizacional (D0) se compone de desempeño financiero (DF) y desempeño no financiero (DnF): el primero comprende el retorno de la inversión, el crecimiento de las ganancias, el crecimiento de las ventas y el aumento de la participación en el mercado (Judge y Douglas, 1998; Richard et al., 2009). Por su parte, el segundo alude al aumento de la satisfacción del cliente, el mejoramiento de la imagen corporativa, el aumento del valor de las marcas y de la productividad de los empleados (Lee et al., 2011).

\subsection{Capacidad de tecnología de la información vs. el DF y el DnF}

En los últimos años, la medición de la rentabilidad que se deriva de las inversiones en TI ha sido una de las principales preocupaciones de los administradores e investigadores (Liu et al., 2008). Brynjolfsson y Hitt (1996) proporcionaron evidencia de una relación positiva entre la inversión en TI y el rendimiento de las empresas, para ello, compararon empresas líderes con empresas seleccionadas deliberadamente que coincidieran con las primeras en tamaño y sector, y se demostró que a mayor capacidad de TI se obtienen mejores rendimientos, resultados que más tarde fueron corroborados por otros investigadores (Bharadwaj, 2000; Santhanam y Hartono, 2003). Sin embargo, aunque la evidencia reciente indica una relación no significativa entre las CTI y el desempeño financiero y no financiero en empresas situadas en países desarrollados en donde se presenta una alta articulación de la TI con los negocios (Chae et al., 2014), en el contexto de un país emergente sí podría seguir siendo una fuente de ventaja competitiva (Liu et al., 2008). 
En ese orden de ideas, las CTI influyen sobre el DF porque facilitan la operación del negocio, debido a que la correcta implementación de una plataforma robusta de TI facilita la realización de tareas y flujos de información de una manera más eficiente, rápida y oportuna, lo cual repercute positivamente sobre la rentabilidad de la organización (Broadbent et al., 2003). Sumado a ello, una operación más eficiente permite fijar precios más competitivos, que se traducen en nuevos clientes, más ventas y por ende más ganancias (Nakata et al., 2008).

Otros autores plantean que las CTI facilitan la tarea de llevar al mercado productos más novedosos, mejorados o difíciles de imitar, e incrementar las ventas de la organización y obtener una mayor retención de sus clientes actuales (Broadbent et al., 2003). Es por esto que una postura proactiva de las CTI permite identificar nuevas TI con el potencial de generar cambios en la estructura de la industria y, por ende, mejorar la participación de mercado de la empresa y consecuentemente obtener un DF superior (Agarwal y Sambamurthy, 2002; Nakata et al., 2008).

En consecuencia, las CTI influyen sobre el DF porque el conocimiento que se captura, almacena y se disemina por medio de las CTI ayuda a mejorar la eficiencia de la empresa, por cuenta de la reducción de los costos operativos de varios procesos organizacionales, lo cual mejora la rentabilidad de la organización. También permite llegar al mercado con productos novedosos y mejorados que marcan una diferencia frente a sus competidores, incrementando significativamente las ventas de la organización. Adicionalmente, las CTI contribuyen a mejorar la calidad de productos y servicios, lo que permite generar una mayor penetración del mercado y aumentar la participación de la empresa. Por lo tanto, se plantea la hipótesis H1a (ver figura 1).

H1a: Las CTI tienen un efecto positivo sobre el DF.

De otra parte, las CTI influyen sobre el DnF porque agregan nuevos atributos a las marcas, asociados a la novedad y la eficiencia, lo cual eleva su valor, puesto que los clientes están dispuestos a pagar más por un producto altamente diferenciado (Shocker et al., 1994). Así mismo, las CTI ayudan al desarrollo de una reputación al permitir la oferta de productos fabricados con tecnología avanzada (Flikkema et al., 2014), el cumplimiento de la entrega de pedidos en un corto plazo, la personalización de los productos, lo cual también aumenta el valor de las marcas de manera significativa (Shocker et al., 1994; Schiffman y Kanuk, 2010; Mohd y Noordin, 2015). 


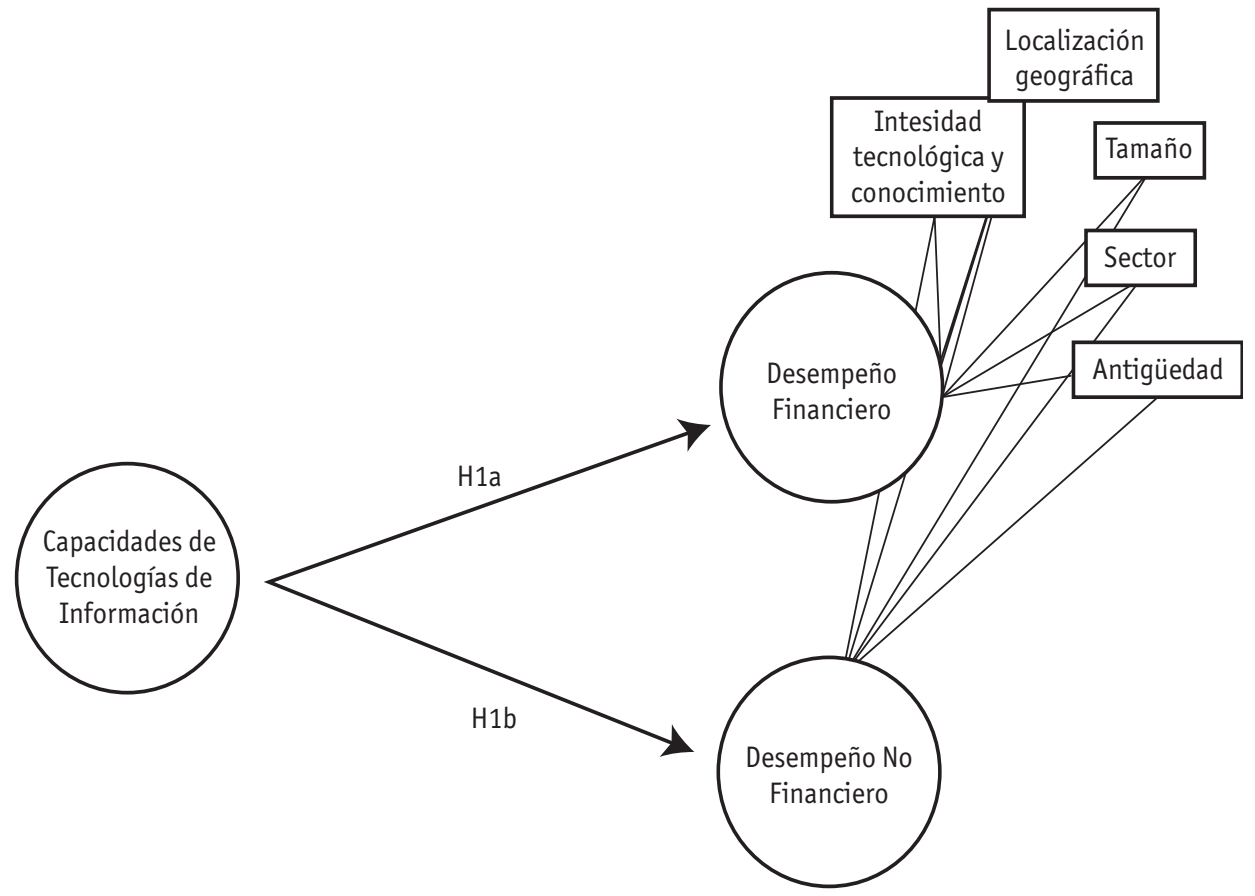

Figura 1. Modelo directo entre CTI, DF y DnF

Fuente: elaboración propia.

De igual manera, cuando el personal interno de la compañía trabaja junto con sus clientes externos dándole un uso eficaz a las TI, puede planificar y desarrollar nuevas aplicaciones tecnológicas dentro de la organización e incorporar el uso de sistemas inteligentes enfocados en la satisfacción del cliente como los CRM, lo cual mejora los procesos de comunicación e incrementa la confianza y la fidelidad de los clientes. De este modo, las CTI se convierten en una fuente de ventajas competitivas, porque sirven de base para ofrecer experiencias únicas y personalizar los productos y servicios (Nakata et al., 2008; Jiao, Chang y Lu, 2008). Las CTI también ayudan a fortalecer la relación con el cliente, porque permiten dinamizar el proceso de resolución de quejas, peticiones y sugerencias, y dar respuestas de forma ágil y oportuna, lo cual incide sobre la satisfacción de los clientes y el valor de las marcas (Nakata et al., 2008).

De otro lado, cuando los empleados utilizan las CTI en sus tareas cotidianas, logran un incremento en la eficiencia, mejoran la planeación y el control, y la efectividad en la toma de decisiones (Dewan, 1997; Lin, 2007). De este modo, también se pueden 
detectar oportunidades de mejora en los procesos internos, lo cual convierte a las CTI en un medio clave para la mejora de la productividad de la empresa (Zhang y Tansuhaj, 2007; Zawislak et al., 2012).

En definitiva, la CTI influye sobre el DnF de diversas maneras. En relación con la satisfacción de los clientes: mejoran los flujos de información que permiten atenderlos de forma ágil y oportuna. En relación con el valor de las marcas: las CTI mejoran la eficiencia interna y facilitan la innovación en productos y procesos, lo cual incide positivamente en la forma en la que los clientes perciben las marcas. En relación con la productividad de los empleados: las CTI aumentan la eficiencia asociada a la realización de las tareas cotidianas, y ayudan a la identificación de oportunidades de mejora de los procesos internos. Por lo tanto, se plantea la siguiente hipótesis H1b (ver figura 1).

H1b: Las CTI tienen un efecto positivo sobre el DnF.

\subsection{Capacidad de absorción (CAB)}

La CAB se entiende como la habilidad de una empresa de reconocer información nueva y externa, asimilarla, transformarla y aplicarla con el fin de crear valor (Cohen y Levinthal, 1990). De acuerdo con Zahra y George (2002), la CAB comprende cuatro dimensiones: la primera es denominada capacidad de adquisición de información, la cual es entendida como la habilidad de la organización para localizar, identificar, evaluar y adquirir conocimiento externo que es importante para el desarrollo de sus operaciones (Lane y Lubatkin, 1998). La segunda dimensión es la capacidad de asimilación, entendida como la destreza de una empresa para comprender el conocimiento externo; es decir, es la capacidad de analizar, clasificar, procesar, interpretar, y en última instancia, internalizar y comprender este conocimiento (Cohen y Levinthal, 1990; Szulanski, 1996). En tercer lugar se tiene la dimensión denominada capacidad de transformación, que se refiere a la habilidad organizacional para facilitar la transferencia y la combinación de los conocimientos previos con los nuevos conocimientos adquiridos o asimilados (Jansen et al., 2005; Todorova y Durisin, 2007). Y la cuarta dimensión es conocida como la capacidad de explotación, la cual no es más que el conjunto de prácticas de una empresa para incorporar los conocimientos adquiridos, asimilarlos y transformarlos en sus operaciones y rutinas para la aplicación y el uso en la organización con fines comerciales (Lane, Salk y Lyles, 2001). 


\subsection{Efectos mediadores de la CAB}

La capacidad de infraestructura de TI proporciona una plataforma de herramientas que provee información para alinear, aumentar eficiencia y optimizar las operaciones del negocio con la finalidad de generar resultados financieros tangibles para la organización como el aumento de las ganancias y la rentabilidad. Sin embargo, para que ello sea posible, es necesaria la intervención de las $C A B$, para que los colaboradores analicen los datos suministrados por las TI y en reuniones debatan y mediante metodologías como lluvias de ideas, propongan estrategias de fijación de precios, posicionamiento de productos, canales de promoción y de distribución (Sáenz et al., 2014), y así combinar el conocimiento nuevo con el existente, y de esta manera orientar el modelo de negocio a la reducción de los costos operativos (Lieberman y Montgomery, 1988; Roberts y Amit, 2003; Sorescu et al., 2007).

En la literatura también se encuentra que las CTI integran conocimientos que le permiten a la organización ofrecer en el mercado productos con un alto nivel de novedad que marcan diferencia frente a la competencia, incrementando el porcentaje de ventas (Liu et al., 2013). Pero esto requiere del soporte de las habilidades propias de la CAB para adquirir e integrar la información proveniente del entorno y a partir de ahí aplicarla en ideas con gran potencial comercial que puedan ser materializadas en proyectos de reingeniería y desarrollo de productos innovadores con funciones avanzadas (Bayus, Erickson y Jacobson, 2003; Srinivasan et al., 2009). De este modo, se puede utilizar la innovación como un medio para hacer frente a las presiones de la competencia, cambiantes demandas de los clientes y la exigencia constante de nuevos y mejores productos y servicios (Govindarajan y Kopalle, 2006; Jansen et al., 2006; Prajogo y Ahmed, 2006).

Lo mismo ocurre en el caso de la incidencia de las CTI en la participación de mercado, por cuenta de la información recopilada por las TI que sirve para ofrecer productos novedosos (Liu et al., 2013). Sin embargo, para ello es preciso hacer uso de las prácticas de las CAB para que la organización logre identificar los aliados estratégicos de los que pueda extraer información valiosa, y entonces contactarlos y establecer rutinas de colaboración y retroalimentación que le permitan explorar el mercado (Daghfous, 2004; Chen et al., 2009), con el propósito de encontrar y explotar oportunidades comercialmente valiosas con determinados estándares de calidad, y así formular prototipos que le faciliten penetrar en nuevos segmentos y lograr la atracción de clientes nuevos y la retención de los actuales (Bayus et al., 2003; Srinivasan et al., 2009; Volberda et al., 2010). Por lo tanto, se plantea la hipótesis H2a (ver figura 2). 
H2a: La relación entre las CTI y el DF esta mediada por la CAB.

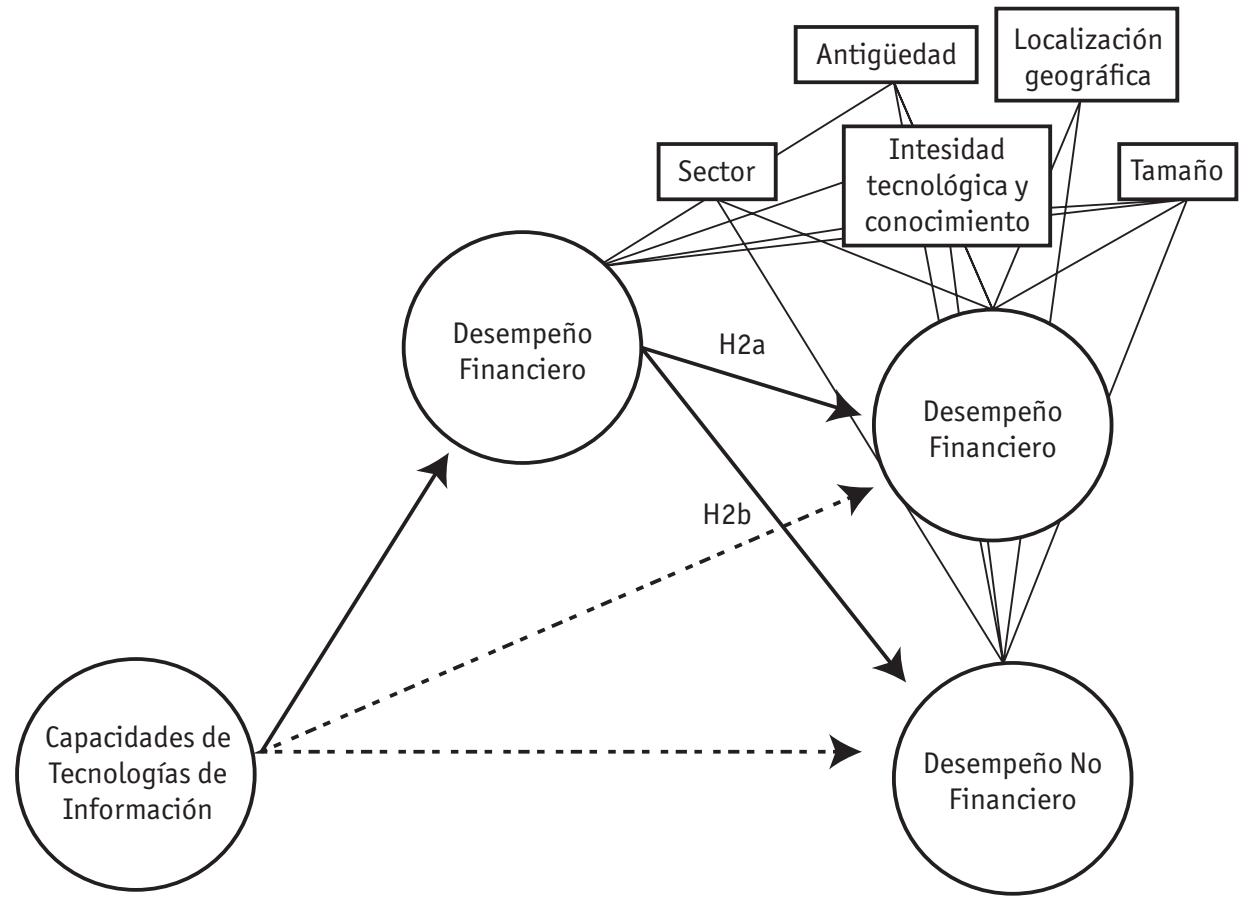

Figura 2. Modelo mediado de CAB en la relación entre CTI, DF y DnF

Fuente: elaboración propia.

De otra parte, las CTI favorecen la respuesta oportuna a peticiones y sugerencias de los clientes, es decir, se fortalecen las relaciones con los clientes, impactando positivamente su grado de satisfacción (Nakata et al., 2008). No obstante, es necesario adoptar rutinas propias de la $C A B$, que permiten adquirir conocimiento nuevo a través del benchmarking en empresas de diversos sectores (incluyendo al que pertenece) y así aprender del know how de las empresas líderes (Selnes y Sallis, 2003; Chen, Lin y Chang, 2009) y aplicarlo en metodologías que permitan la confirmación temprana de pedidos, quejas y reclamos, lo cual genera agilidad en los tiempos de respuesta y de esta manera obtener un mejoramiento significativo en la calidad y el nivel de servicio al cliente y demás aspectos que determinan la satisfacción del mismo (Hamel, 1991; Zahra y George, 2002).

Asimismo, las CTI proporcionan canales que facilitan una comunicación permanente con el consumidor final y en esa medida permiten identificar las necesidades y los deseos 
de su mercado, lo cual favorece el posicionamiento y el valor de la marca (Jansen et al., 2006; Prajogo y Ahmed, 2006). Sin embargo, para que esto sea posible se requiere de la convergencia de la $C A B$, para que las compañías recopilen información a partir de encuestas y a partir de dichos datos generar campañas publicitarias (Schiffman y Kanuk, 2010), invertir en cambio y/o rediseño de logotipo o eslogan, para lograr recordación (Jansen et al., 2006; Chen y Huang, 2009; Lichtenthaler, 2009).

Finalmente, las CTI influyen positivamente en la productividad de los empleados al permitir la comunicación entre los colaboradores, no obstante, es de vital importancia la intervención de las habilidades de la $C A B$, que proporciona ambientes en los cuales el personal se motive y desarrolle el hábito de compartir información útil y aplicable que procede del entorno, de manera que se mejore la agilidad en las relaciones intraorganizacionales mediante el establecimiento de redes formales e informales para la transferencia de conocimiento, lo que maximiza la amplitud y la calidad de los intercambios de información entre las diferentes áreas funcionales, y así impactar positivamente los procesos de aprendizaje al interior de la organización, la toma de decisiones conjunta y el rediseño de procesos (George et al., 2001; Zahra y George, 2002; Lane et al., 2006; Laursen y Salter, 2006; Ferreras-Méndez et al., 2015). De acuerdo con lo anterior, se plantea la hipótesis H2b (ver figura 2).

H2b: La relación entre las CTI y el DnF está mediada por la CAB.

\section{Metodología}

\subsection{Muestra y recolección de los datos}

El modelo de hipótesis representado en la figura 2 se contrastó en una muestra de empresas ubicadas en Colombia, país de economía emergente y tecnológicamente seguidor (Hoskisson et al., 2000; Castellacci, 2001), ocupa el puesto 89 en el ranking relacionado con el Nivel de Absorción Tecnológica de las Empresas, el 51 en el desarrollo de nuevos productos y servicios, y el 54 por el impacto de las CTI en nuevos modelos organizacionales, dentro de una lista de 143 países (WEF, 2015). Por otra parte, según el Índice de desarrollo de las TIC 2015, Colombia se ubicó en el puesto 75, por encima de países de la región como Panamá que ocupó el puesto 89; Ecuador, el 90; México, el 95 y Perú, el 104 (UIT, 2015). De igual modo los indicadores oficiales respecto al sector TIC en Colombia entre el 2009 y 2013 presentan un incremento del $32 \%$ en el valor agregado (MinTIC, 2015). 
La muestra está conformada por empresas manufactureras y de servicios, la cual contiene sectores de uso intensivo de TIC (DANE, 2014) tanto en PyMEs como grandes empresas (tabla 1), entre los que se destacan el sector de actividades de administración empresarial, y el sector de información y comunicaciones. Por otra parte, los datos se obtuvieron durante el trabajo de campo realizado en octubre de 2015, mediante el envío del cuestionario por correo electrónico a personal directivo o de nivel estratégico de diferentes áreas funcionales de cada una de las empresas consultadas (tabla 2). Finalmente, se obtuvieron 102 respuestas válidas, lo cual permite garantizar una potencia satisfactoria, superior al 80\% (Cohen, 1992; Hair et al., 2014).

\section{Tabla 1}

Características de las empresas de la muestra

\begin{tabular}{|c|c|c|}
\hline Sector & Frecuencia & Porcentaje (\%) \\
\hline Actividades de administración empresarial; actividades de consultoría de gestión & 23 & 22,55 \\
\hline Información y comunicaciones & 16 & 15,69 \\
\hline Educación & 12 & 11,76 \\
\hline Confección de prendas de vestir & 8 & 7,84 \\
\hline Actividades de atención de la salud humana y de asistencia social & 6 & 5,88 \\
\hline Alojamiento y servicios de comida & 6 & 5,88 \\
\hline $\begin{array}{l}\text { Comercio al por mayor y al por menor, reparación de vehículos automotores y } \\
\text { motocicletas }\end{array}$ & 5 & 4,90 \\
\hline Fabricación de productos elaborados de metal, excepto maquinaria y equipo & 4 & 3,92 \\
\hline Fabricación de sustancias y productos químicos & 4 & 3,92 \\
\hline Otras actividades manufactureras & 4 & 3,92 \\
\hline Transporte y almacenamiento & 3 & 2,94 \\
\hline Fabricación de aparatos y equipo eléctrico & 2 & 1,96 \\
\hline $\begin{array}{l}\text { Fabricación de instrumentos, aparatos y materiales médicos y odontológicos } \\
\text { (incluido mobiliario) }\end{array}$ & 2 & 1,96 \\
\hline Fabricación de juegos, juguetes y rompecabezas & 1 & 0,98 \\
\hline Actividades financieras y de seguros & 1 & 0,98 \\
\hline Actividades inmobiliarias & 1 & 0,98 \\
\hline $\begin{array}{l}\text { Distribución de agua, evacuación y tratamiento de aguas residuales, gestión } \\
\text { de desechos y actividades }\end{array}$ & 1 & 0,98 \\
\hline Otras actividades de servicios & 3 & 2,94 \\
\hline Tamaños por número de empleados & Frecuencia & Porcentaje \\
\hline Grandes empresas & 25 & 24,51 \\
\hline Pymes & 77 & 75,49 \\
\hline
\end{tabular}

Fuente: elaboración propia. 


\section{Tabla 2}

Área funcional de la persona encuestada

\begin{tabular}{lcc}
\hline \multicolumn{1}{c}{ Área funcional del encuestado } & Frecuencia & Porcentaje (\%) \\
\hline Presidencia o gerencia general & 51 & 50,00 \\
Sistemas y tecnología & 14 & 13,73 \\
Mercadeo & 12 & 11,76 \\
Otros & 8 & 7,84 \\
Investigación y desarrollo & 6 & 5,88 \\
Finanzas & 5 & 4,90 \\
Producción & 3 & 2,94 \\
Recursos humanos & 3 & 2,94 \\
\hline
\end{tabular}

Fuente: elaboración propia.

\subsection{Escalas de medida}

El constructo CTI se midió haciendo uso de la escala propuesta por Lu y Ramamurthy (2011), que comprende once ítems (ver anexo). En este caso se utilizó una escala de Likert con cinco opciones de respuesta, que va desde muy inferior en comparación con otras empresas del sector (1) hasta muy superior en comparación con otras empresas del sector (5) para los ítems del 1 al 7; y desde totalmente de acuerdo (1), hasta totalmente en desacuerdo (5) para los ítems del 8 al 11. Por su parte, la CAB se midió con la escala propuesta por Flatten et al. (2011), compuesta por catorce ítems (ver anexo), también se utilizó una escala de Likert que va desde totalmente de acuerdo (1), hasta totalmente en desacuerdo (5).

En cambio, para la medición del DF se utilizó la escala propuesta por Judge y Douglas (1998), y para el DnF la desarrollada por Lee et al. (2011); en ambos casos se emplearon cuatro ítems (ver anexo), y se utilizó una escala Likert que va desde muy inferior en comparación con otras empresas del sector (1), hasta muy superior en comparación con otras empresas del sector (5) (ver anexo).

\subsection{Análisis de los datos}

\subsubsection{VARIANZA DEL MÉTODO COMÚN}

Respecto a las estrategias para evitar el problema de la varianza del método común se adoptaron varias medidas relacionadas con el diseño del formulario de encuesta y la 
toma de los datos tales como garantizar el anonimato de las empresas y personas que respondieron la encuesta y la confidencialidad de la información suministrada, y mezclar las preguntas en el formulario (Chang et al., 2010; Conway y Lance, 2010). Además, la prueba del factor simple de Harman confirma que los datos recolectados tienen la varianza explicada por el primer factor del 47,75\%, lo cual disminuye la posibilidad de que los datos presenten el problema en mención (Podsakoff et al., 2003).

\subsubsection{FIABILIDAD Y VALIDEZ}

Para el análisis del modelo de medición reflectivo se utilizó el enfoque de mínimos cuadrados parciales, en el software SmartPLS 2.0 (Henseler et al., 2009). La fiabilidad individual para cada ítem de la escala de medida se verificó siendo cada carga factorial superior a 0,7 (Hair et al., 2014). También se constató que el índice de fiabilidad compuesta para cada constructo es superior a 0,7 (Chi et al., 2010). Asimismo, se evaluó la validez convergente mediante el índice de Varianza Extraída Promedio (AVE), cuyo valor mínimo recomendado según la literatura es de 0,5 (Fornell y Larcker, 1981; Hair et al., 2014), encontrándose que todos los constructos de este estudio tienen un AVE mayor al mínimo requerido de 0,5 (ver tabla 3).

\section{Tabla 3}

Fiabilidad y validez convergente

\begin{tabular}{cccccc}
\hline Constructos & $\begin{array}{c}\text { Carga } \\
\text { estandarizada }\end{array}$ & Error estándar & Valort & $\begin{array}{c}\text { Fiabilidad } \\
\text { compuesta }\end{array}$ & $\begin{array}{c}\text { Índice de varianza } \\
\text { extraída }\end{array}$ \\
\hline CTI & & & & 0,95 & 0,64 \\
CTI1 & $0,79^{*}$ & 0,06 & 12,62 & & \\
CTI2 & $0,89^{*}$ & 0,02 & 41,58 & & \\
CTI3 & $0,89^{*}$ & 0,02 & 50,54 & & \\
CTI4 & $0,78^{*}$ & 0,05 & 16,04 & \\
CTI5 & $0,80^{*}$ & 0,04 & 20,65 & \\
CTI6 & $0,78^{*}$ & 0,05 & 15,35 & & \\
CTI7 & $0,79^{*}$ & 0,05 & 17,43 & & \\
CTI8 & $0,79^{*}$ & 0,04 & 17,87 & & \\
CTI9 & $0,77^{*}$ & 0,05 & 15,41 & & \\
CTI10 & $0,78^{*}$ & 0,05 & 16,65 & & \\
CTI11 & $0,71^{*}$ & 0,08 & 9,40 & & Continúa $\rightarrow$ \\
DF & & & & 0,92 & \\
\hline
\end{tabular}




\begin{tabular}{cccccc}
\hline Constructos & $\begin{array}{c}\text { Carga } \\
\text { estandarizada }\end{array}$ & Error estándar & Valort & $\begin{array}{c}\text { Fiabilidad } \\
\text { compuesta }\end{array}$ & $\begin{array}{c}\text { Índice de varianza } \\
\text { extraída }\end{array}$ \\
\hline DF1 & $0,88^{*}$ & 0,04 & 21,78 & \\
DF2 & $0,88^{*}$ & 0,04 & 21,33 & \\
DF3 & $0,87^{*}$ & 0,04 & 21,49 & \\
DF4 & $0,82^{*}$ & 0,07 & 12,24 & & \\
DnF & & & & 0,89 \\
DnF1 & $0,76^{*}$ & 0,07 & 10,69 & \\
DnF2 & $0,88^{*}$ & 0,04 & 24,01 & \\
DnF3 & $0,88^{*}$ & 0,03 & 31,13 & \\
DnF4 & $0,76^{*}$ & 0,07 & 11,42 & \\
CAB & & & & \\
CAB1 & $0,75^{*}$ & 0,06 & 12,83 & \\
CAB2 & $0,80^{*}$ & 0,05 & 15,45 & \\
CAB3 & $0,76^{*}$ & 0,05 & 14,95 & \\
CAB4 & $0,81^{*}$ & 0,04 & 20,79 & \\
CAB5 & $0,82^{*}$ & 0,04 & 20,83 & \\
CAB6 & $0,77^{*}$ & 0,05 & 16,24 & \\
CAB7 & $0,76^{*}$ & 0,06 & 13,70 & \\
CAB8 & $0,81^{*}$ & 0,05 & 18,08 & \\
CAB9 & $0,76^{*}$ & 0,06 & 12,31 & \\
CAB10 & $0,80^{*}$ & 0,05 & 15,31 & \\
CAB11 & $0,69^{*}$ & 0,06 & 12,44 & \\
CAB12 & $0,77^{*}$ & 0,05 & 16,52 & \\
CAB13 & $0,82^{*}$ & 0,04 & 19,76 & \\
CAB14 & $0,82^{*}$ & 0,04 & 21,03 & \\
\\
\hline
\end{tabular}

\begin{tabular}{cccc}
\hline & \multicolumn{3}{c}{ Variables de Control } \\
\hline Antigüedad & 1 & 0 & 0 \\
\hline Región & 1 & 0 & 0 \\
\hline Sector & 1 & 0 & 0 \\
\hline Tamaño & 1 & 0 & 0 \\
\hline $\begin{array}{c}\text { Intensidad tecnológica } \\
\text { y de conocimiento }\end{array}$ & 1 & 0 & 0 \\
\hline
\end{tabular}

${ }^{*} \mathrm{p} \leq 0,001$

Fuente: elaboración propia. 


\subsubsection{VALIDEZ DISCRIMINANTE}

Para garantizar que cada constructo es único e independiente el uno del otro, y que además captura un fenómeno no presentado por otro constructo, el análisis factorial confirmatorio arrojó resultados que permitieron confirmar la validez discriminante, dado que la raíz cuadrada del índice de la varianza extraída para cada uno de los constructos es superior a las correlaciones entre cada uno de los distintos constructos (Chin, 1998). En la tabla 4 se observa que esta condición se cumple en cada uno de los casos.

Tabla 4

Validez discriminante

\begin{tabular}{ccccc}
\hline Constructos & CTI & DF & DnF & CAB \\
\hline CTI & 0,799 & & & \\
DF & 0,495 & 0,863 & & \\
DnF & 0,566 & 0,631 & 0,823 & \\
CAB & 0,681 & 0,516 & 0,579 & 0,782 \\
\hline
\end{tabular}

Fuente: elaboración propia.

\subsubsection{VARIABLES DE CONTROL}

Sumado a lo anterior, en este estudio se incluyeron variables de control para evitar interferencias y desviaciones en los resultados; para ello se consideraron el tamaño, el sector, la intensidad tecnológica del sector, la antigüedad y la región en la que se encuentra ubicada la empresa, dado que investigaciones previas han reportado la incidencia de algunas de ellas (Francalanci y Morabito, 2008; Nakata et al., 2008; Lu y Ramamurthy, 2011; Liu et al., 2013). Las variables de control se incorporaron al estudio de la siguiente manera:

1. Tamaño: esta variable corresponde al logaritmo natural de los números de empleados de las empresas.

2. Sector dummy: el cero (0) representa las empresas del sector manufacturero y el uno (1) empresas del sector servicios

3. Localización geográfica dummy: el cero (0) agrupa las empresas ubicadas fuera de Antioquia y Bogotá y el uno (1) las ubicadas en Antioquia y Bogotá.

4. Antigüedad: se mide la edad de la empresa con el logaritmo natural de los años de antigüedad de las empresas. 
5. Intensidad tecnológica y de conocimiento: para el caso de sector manufacturero " 0 " agrupa empresas de baja y media - baja intensidad de tecnología y el " 1 " empresas de media alta y alta intensidad de tecnología. Para el caso de sector servicios " 0 " agrupa empresas de baja intensidad de conocimiento y " 1 " empresas de alta intensidad de conocimiento (Eurostat, 2009).

\subsubsection{TEST DEL EFECTO MEDIADOR}

Para verificar el efecto mediador se determinaron las trayectorias de los dos modelos de hipótesis, directo y mediado, calculando el nivel de significancia estadística de los coeficientes en cada una de las relaciones del modelo empleando el método de los mínimos cuadrados parciales (Baron y Kenny, 1986), cuya aplicación implica ejecutar un procedimiento de remuestreo o bootstraping de 5.000 submuestras con el fin de obtener los valores $t$ y los errores estándar de los coeficientes de cada una de las trayectorias (Henseler et al., 2009).

Asimismo, para confirmar de manera concluyente la existencia y significancia del efecto mediador de la CAB en la relación entre CTI, DFy DnF, se efectuó el análisis bootstrap-percentil (Preacher y Hayes, 2008; Williams y MacKinnon, 2008), que implica calcular el producto de los efectos indirectos con los datos el remuestreo y generar un intervalo de confianza del $95 \%$. En caso de existir el efecto mediador, se calcula la magnitud de la mediación mediante la Varianza Explicada (VAF por sus siglas en inglés), la cual indica que no existe mediación si VAF es menor que 20. Por otro lado, cuando $20<\mathrm{VAF}<80$ la mediación es parcial, es decir, la variable independiente todavía tiene un efecto directo significativo sobre la dependiente; por último si VAF es mayor que 80, existe una relación de mediación total (Hair et al., 2014).

\subsection{Predictibilidad y relevancia predictiva}

Por otro lado, se verificó el coeficiente $\mathrm{R}^{2}$ de los constructos endógenos con la finalidad de evaluar la predictibilidad del modelo directo y del modelo mediado. En todos los casos superó el valor mínimo de 0,1 (Falk y Miller, 1992). Sumado a lo anterior, se observó que el modelo mediado explica el $42,5 \%$ de la varianza del DF y $42,7 \%$ de la varianza del $\mathrm{DnF}$, representando estos resultados un nivel de predictibilidad satisfactorio. También se examinaron los valores $Q^{2}$ para los constructos dependientes y se verificó que los datos fueran superiores a cero (0), con esto se confirma la existencia de la característica de relevancia predictiva en los dos modelos de hipótesis (Hair et al., 2014). 


\section{Resultados}

Los análisis estadísticos se ejecutaron mediante el empleo de la metodología de mínimos cuadrados parciales y se efectuaron sobre la estructura de las relaciones planteadas en de H1a, H1b, H2a y H2b. En el caso del modelo directo, en la tabla 5 se muestra que la relación entre CTI influye de manera positiva sobre el $D F(\beta=0,46$, valor $t=5,39)$ y el $\operatorname{DnF}(\beta=0,55$, valor $t=6,26)$; por ende se aceptan H1a y H1b. Por otro lado, en el modelo mediado estas relaciones dejan de ser significativas $(\beta=0,09$, valor $t=0,67)$ para el $D F y$ para el $\operatorname{DnF}(\beta=0,26$, valor $t=1,95)$. En cambio, se encontró que la relación entre CTI y CAB es significativa $(\beta=0,78$, valor $t=13,18)$ así como también la relación entre $C A B$ y $D F(\beta=0,46$, valor $t=3,40)$ y $C A B$ y $\operatorname{DnF}(\beta=0,37$, valor $t=2,85)$. Con base en lo anterior se aceptan $\mathrm{H} 2 \mathrm{a}$ y $\mathrm{H} 2 \mathrm{~b}$. Sumado a ello, las variables de control no ejercen relaciones significativamente influyentes en los constructos del modelo.

\section{Tabla 5}

Resultados de las ecuaciones estructurales

\begin{tabular}{|c|c|c|c|}
\hline Modelo & Trayectorias & Coeficiente & Valor $\mathrm{t}$ \\
\hline \multirow{13}{*}{ Directo } & $\begin{array}{l}\mathrm{CTI} \rightarrow \mathrm{DF} \\
\left(\mathrm{R}^{2}=0,33 \mathrm{Q}^{2}=0,25\right) ;\end{array}$ & 0,46 & 5,39 \\
\hline & $\begin{array}{l}\mathrm{CTI} \rightarrow \mathrm{DnF} \\
\left(\mathrm{R}^{2}=0,37 ; \mathrm{Q}^{2}=0,24\right) ;\end{array}$ & 0,55 & 6,26 \\
\hline & Variables & & \\
\hline & Antigüedad $\rightarrow$ DF & 0,04 & 0,40 \\
\hline & Antigüedad $\rightarrow$ DnF & 0,12 & 1,02 \\
\hline & Localización geográfica $\rightarrow$ DF & $-0,23$ & 2,41 \\
\hline & Localización geográfica $\rightarrow$ DnF & $-0,19$ & 1,80 \\
\hline & Sector $\rightarrow$ DF & $-0,09$ & 0,84 \\
\hline & Sector $\rightarrow$ DnF & 0,02 & 0,22 \\
\hline & Tamaño $\rightarrow$ DF & 0,14 & 1,22 \\
\hline & Tamaño $\rightarrow$ DnF & $-0,14$ & 1,18 \\
\hline & Intensidad tecnológica y de conocimiento $\rightarrow$ DF & 0,01 & 0,05 \\
\hline & Intensidad tecnológica y de conocimiento $\rightarrow \mathrm{DnF}$ & 0,07 & 0,73 \\
\hline \multirow{4}{*}{ Mediado } & $\mathrm{CTI} \rightarrow \mathrm{DF}$ & 0,09 & 0,67 \\
\hline & $\mathrm{CTI} \rightarrow \mathrm{DnF}$ & 0,26 & 1,95 \\
\hline & $\begin{array}{l}\mathrm{CTI} \rightarrow \mathrm{CAB} \\
\left(\mathrm{R}^{2}=0,56 ; \mathrm{Q}^{2}=0,27\right)\end{array}$ & 0,78 & 13,18 \\
\hline & $\begin{array}{l}C A B \rightarrow D F \\
\left(R^{2}=0,42 ; Q^{2}=0,32\right) ;\end{array}$ & 0,46 & 3,40 \\
\hline
\end{tabular}




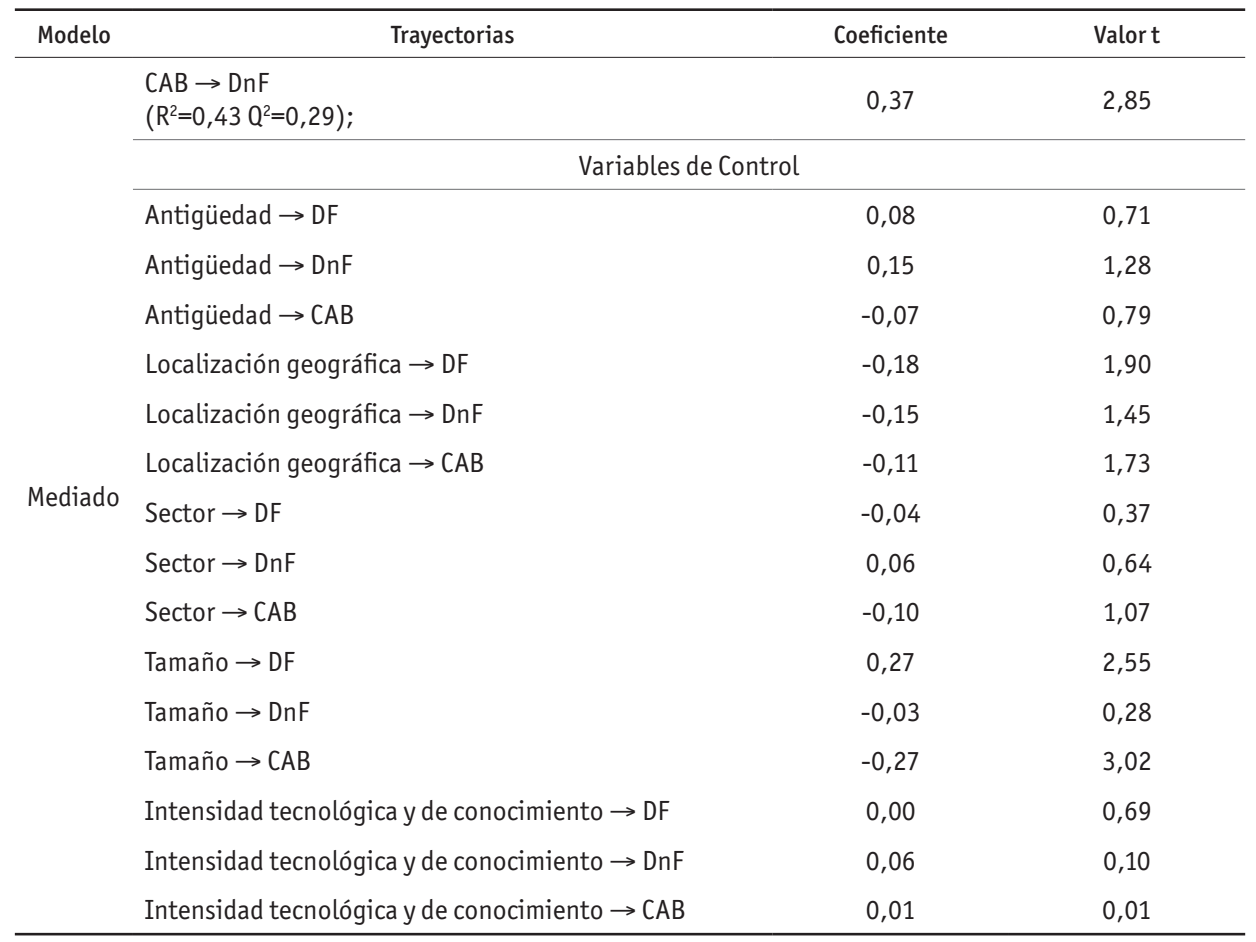

Fuente: elaboración propia.

Ahora bien, la ejecución de la prueba del bootstrap percentil que se efectuó con un intervalo de confianza del 95\%, implica calcular el producto de los efectos indirectos con los datos del remuestreo. En la tabla 6 se visualiza que los intervalos calculados para el DF y el DnF no contiene el valor cero (0), lo que sugiere que el efecto mediador planteado en la hipótesis $\mathrm{H} 2 \mathrm{a}$ y $\mathrm{H} 2 \mathrm{~b}$ es significativo.

\section{Tabla 6}

Test del efecto mediador DF y DnF

\begin{tabular}{cccccccc}
\hline \multirow{2}{*}{ Constructo } & \multicolumn{2}{c}{ Efecto total } & \multicolumn{2}{c}{ Efecto directo } & $\begin{array}{c}\text { Efecto } \\
\text { indirecto }\end{array}$ & $\begin{array}{c}\text { Bootstrap Percentil/Intervalo de } \\
\text { confianza (95\%) }\end{array}$ \\
\cline { 2 - 7 } & Coeficiente & Valort & Coeficiente & Valort & Coeficiente & Límite inferior & Límite superior \\
\hline DF & 0,46 & 5,39 & 0,09 & 0,67 & 0,36 & 0,14 & 0,60 \\
DnF & 0,55 & 6,26 & 0,26 & 1,95 & 0,29 & 0,09 & 0,52 \\
\hline
\end{tabular}

Fuente: elaboración propia. 
Para dar continuidad al análisis de la mediación se calculó el valor de VAF, el cual es de $80,4 \%$ para el DF y de 53,2\% para el DnF, lo que indica que la mediación es total para el primer constructo y parcial para el segundo, por estar en el intervalo de $20 \%$ a $80 \%$. Esta proporción establece la magnitud del efecto indirecto sobre el efecto total (Hair et al., 2014). En definitiva, se aceptan H1a, H1b, H2a y H2b.

Estos hallazgos son de gran importancia porque indican que cualquier inversión en TI, que apunte al desarrollo de la capacidad de TI, si no está acompañada del desarrollo de una capacidad blanda como la absorción de conocimiento, tiene un alto riesgo de fracaso por la incapacidad organizacional de articular las TI con el conocimiento existente y de generar valor con base en este recurso estratégico.

\section{Conclusiones}

Este artículo aporta evidencia empírica concluyente con relación al impacto de las CTI sobre el DFy el DnF, el cual es significativamente positivo, en contraste con la controversia existente en torno a esta relación. Los resultados que se presentan en este artículo aportan claridad a la discusión acerca del impacto de las CTI sobre el DF y el DnF, por lo menos en el contexto de una economía emergente que ocupa una posición intermedia en el ranking del Reporte Global de Tecnologías de la Información. Además la metodología empleada supera algunas limitaciones presentadas en trabajos previos, entre las cuales está el incluir en el estudio más variables de control como la intensidad tecnológica y de conocimiento, y el emplear una muestra que abarcara todas las áreas funcionales de las organizaciones que participaron en la investigación.

Otra contribución académica es que se logró demostrar la mediación de la CAB, en particular se encontró que existe una mediación total en la relación de las CTI y el DF y parcial en el caso de las CTI y el DnF. De esta forma, queda demostrado que las CTI son un constructo clave, pero si no se combina con habilidades blandas como las CAB, su efecto y retorno sobre el DF y el DnF puede ser imperceptible y poco representativo. Este resultado se suma a otros hallazgos de la literatura que vienen demostrando que la incidencia es insuficiente si no se articula con habilidades de un corte estratégico, en este caso con la $C A B$, la cual indiscutiblemente muestra un efecto mediador, perfilándose como uno de los principales constructos mediadores en el impacto que las CTI ejerce sobre otros constructos organizacionales, en este caso, con el DF y el DnF. 
De este trabajo se logran identificar algunas implicaciones en la práctica organizacional, concretamente en lo que se refiere a la CAB que como habilidad organizacional potencializa el impacto de las CTI, por lo que los directivos deben implementar rutinas organizacionales tales como motivar a los empleados a utilizar fuentes de información y realizar búsqueda de datos relevantes sobre el sector al que pertenece la empresa y acerca de otras industrias con proveedores, clientes, competidores, universidades, instituciones de investigación, revistas especializadas, conferencias, entre otros. En este sentido, también se debe promover la ejecución de reuniones periódicas entre las diferentes áreas funcionales para intercambiar dicha información, nuevos desarrollos, problemas y logros, que permitan reevaluar las tecnologías existentes y adaptar de manera novedosa las nuevas, de manera que se facilite la toma de decisiones, se logre flexibilidad de la estrategia organizacional y se adquiera la capacidad de dar respuesta rápida a las cambiantes necesidades del mercado, ayudando a la organización a mantener la sostenibilidad de su ventaja competitiva.

De otra parte, en el desarrollo de esta investigación se encontró como limitante la generalización de los resultados, los cuales están supeditados al contexto en el cual se recolectó la información empírica, de tal suerte que Colombia es un país emergente en el que la penetración de las tecnologías de información se encuentran en una posición intermedia a la luz del ranking de disponibilidad de TI (WEF, 2015), la cual es similar a la de la gran mayoría de países emergentes. En comparación con países desarrollados, Colombia presenta ciertas deficiencias en la producción de tecnología y el acceso a los recursos críticos para la generación de innovación, por ejemplo, la infraestructura de la tecnología, la creación de redes eficaces con instituciones de investigación en todo el mundo, entre otras.

Para futuras investigaciones es importante considerar otras variables mediadoras junto con una variable moderadora y extender el modelo a otras líneas donde se evalúe el impacto de las CTI sobre otros constructos internos de naturaleza blanda similares al objeto de estudio de esta investigación, tales como capacidad de innovación, creatividad organizacional (Tiwana y McLean, 2005) y orientación estratégica (Wales et al., 2013). Además, sería interesante establecer la mediación de constructos externos como la innovación abierta (Hung y Chou, 2013) y la capacidad de absorción de la competencia (Hurmelinna y Olander, 2014) sobre la relación entre CTI, DF y DnF para sobrepasar el límite de considerar sólo capacidades internas de la organización. 


\section{Referencias}

Agarwal, R. \& Sambamurthy, V. (2002). Principles and models for organizing the IT function. MIS Quarterly Executive, 1(1), 1-16.

Baron, R. M. \& Kenny, D. A. (1986). The moderator - mediator variable distinction in social psychological research: Conceptual, strategic, and statistical considerations. Journal of Personality and Social Psychology, 51(6), 1173-1182.

Bayus, B., Erickson, G. \& Jacobson, R. (2003). The financial rewards of new product introductions. Management Science, 49(2), 197-210. https://doi.org/10.1287/mnsc.49.2.197.12741

Bharadwaj, A. S. (2000). A resource-based perspective on information technology capability and firm performance: An empirical investigation. MIS Quarterly, 24(1), 169-196. https://doi: 10.2307/3250983

Broadbent, M., McDonald, M. \& Hunter, R. (2003). Does it matter? An HBR Debate. Letters to the editor. Harvard Business Review, 81, 6-10.

Brynjolfsson, E. \& Hitt, L. (1996). Paradox lost? Firm-level evidence on the returns to information systems spending. Management Science, 42(4), 541-558. https://doi.org/10.1287/ mnsc.42.4.541

Brynjolfsson, E. \& Hitt, L. (1997). Breaking boundaries. Information Week, Special Issue, 34-36.

Brynjolfsson, E. \& Hitt, L. (2000). Beyond computation: Information technology, organizational transformation and business performance. Journal of Economic Perspectives, 14(4), 23-48. https://doi: 10.1257/jep.14.4.23

Camisón, C. \& Villar-López, A. (2014). Organizational innovation as an enabler of technological innovation capabilities and firm performance. Journal of Business Research, 67(1), 2891-2902. https://doi.org/10.1016/j.jbusres.2012.06.004

Castellacci, F. (2001). Closing the technology gap? Review of Development Economics, 15(1), 180-197. https://doi.org/10.1111/j.1467-9361.2010.00601.x

Chae, H., Koh, C. \& Prybutok, V. (2014). Information technology capability and firm performance: Contradictory findings and their possible causes. MIS Quarterly, 38(1), 305-326. https://doi:10.25300/MISQ/2014/38.1.14

Chang, S., Van Witteloostuijn, A. \& Eden, L. (2010). From the editors: Common method variance in international business research. Journal of International Business Studies, 41(2), 178-184. https://doi.org/10.1057/jibs.2009.88

Chen, C. \& Huang, J. (2009). Strategic human resource practices and innovation performance the mediating role of knowledge management capacity. Journal of Business Research, 62(1), 104-114. https://doi.org/10.1016/j.jbusres.2007.11.016

Chen, Y., Lin, M. \& Chang, C. (2009). The positive effects of relationship learning and absorptive capacity on innovation performance and competitive advantage in industrial markets. 
Industrial Marketing Management, 38(2), 152-158. https://doi.org/10.1016/j.indmarman.2008.12.003

Chi, M., Zhao, J., Lu, Z. \& Liu, Z. (2010). Analysis of E-business capabilities and performance: From e-SCM process view. In Computer Science and Information Technology (ICCSIT), $3^{\text {rd }}$ IEEE International Conference on (v. 1, pp. 18-22). IEEE. https://doi.org/10.1109/ ICCSIT.2010.5563863

Chin, W. (1998). The partial least squares approach to structural equation modeling. Modern methods for business research, 295(2), 295-336.

Cohen, J. (1992). A power primer. Psychological bulletin, 112(1), 155-159. http://dx.doi. org/10.1037/0033-2909.112.1.155.

Cohen, W. \& Levinthal, D. (1990). Absorptive capacity: A new perspective on learning and innovation. Administrative Science Quarterly, 35(1), 128-152. https://doi.org/10.1016/ B978-0-7506-7223-8.50005-8

Conway, J. \& Lance, C. (2010). What reviewers should expect from authors regarding common method bias in organizational research. Journal of Business and Psychology, 25(3), 325334. https://doi.org/10.1007/s10869-010-9181-6

Daghfous, A. (2004). Absorptive capacity and the implementation of knowledge intensive best practices. S.A.M. Advanced Management Journal, 69(2), 21-27.

Datta, A. (2012). IT-Based knowledge capability and commercialization of innovations: modeling the impacts of ambidexterity and absorptive capacity. International Journal of Knowledge Management, 8(3), 84-98.

Dedrick, J., Gurbaxani, V. \& Kraemer, K. (2003). Information technology and economic performance: A critical review of the empirical evidence. ACM Computing Surveys, 35(1), 1-28. https://doi.org/10.1145/641865.641866

Departamento Administrativo Nacional de Estadística. (2014). Indicadores básicos de tenencia y uso de las tecnologías de la información y la comunicación en empresas. Bogotá: DANE.

Dewan, S. \& Min, C. (1997). The substitution of information technology for other factors of production: A firm level analysis. Management Science, 43(12), 1660-1675.

Eurostat (2009). 'High-technology' and 'knowledge based services' aggregations based on NACE Rev. 2. Eurostat.

Fahy, J. \& Hooley, G. (2002). Sustainable competitive advantage in electronic business: Towards a contingency perspective on the resource-based view. Journal of Strategic Marketing, 10(4), 241-253. https://doi.org/10.1080/0965254022000014532

Falk, R. \& Miller, N. (1992). A primer for soft modeling. University of Akron Press.

Ferreras-Méndez, J., Newell, S., Fernández-Mesa, A. \& Alegre, J. (2015). Depth and breadth of external knowledge search and performance: The mediating role of absorptive capac- 
ity. Industrial Marketing Management, 47, 86-97. https://doi.org/10.1016/j.indmarman.2015.02.038

Flatten, T., Engelena, A., Zahra, S. \& Brettel, M. (2011). A measure of absorptive capacity: Scale development and validation. European Management Journal, 29(2), 98-116. https:// doi.org/10.1016/j.emj.2010.11.002

Flikkema, M., De Man, A. \& Castaldi, C. (2014). Are trademark counts a valid indicator of innovation? Results of an in-depth study of new benelux trademarks filed by SMEs. Industry and Innovation, 21(4), 310-331. https://doi.org/10.1080/13662716.2014.934547

Fornell, C. \& Larcker, D. (1981). Evaluating structural equation models with unobservable variables and measurement error. Journal of Marketing Research, 18(1), 39-50. https:// doi.org/10.2307/3151312

Francalanci, C. \& Morabito, V. (2008). IS integration and business performance: The mediation effect of organizational absorptive capacity in SMEs. Journal of Information Technology, 23(4), 297-312. https://doi.org/10.1057/jit.2008.18

George, G., Zahra, S., Wheatley, K. \& Khan, R. (2001). The effects of alliance portfolio characteristics and absorptive capacity on performance. A study of biotechnology firms. Journal of High Technology Management Research, 12(2), 205-226. https://doi.org/10.1016/ S1047-8310(01)00037-2

Govindarajan, V. \& Kopalle, P. (2006). Disruptiveness of innovations: Measurement and an assessment of reliability and validity. Strategic Management Journal, 27(2), 189-199. https://doi.org/10.1002/smj.511

Hair, J., Hult, G., Ringle, C. \& Sarstedt, M. (2014). Partial least squares structural equation modeling (PLS-SEM) An emerging tool in business research. European Business Review, 26(2), 106-121. https://doi.org/10.1108/EBR-10-2013-0128

Hamel, G. (1991). Competition for competence and interpartner learning within international strategic alliances. Strategic Management Journal, 12(S1), 83-103. https://doi. org/10.1002/smj.4250120908

Henseler, J., Ringle, C. \& Sinkovics, R. (2009). The use of partial least squares path modeling in international marketing. Advances in international marketing, 20(1), 277-319. https:// doi.org/10.1108/S1474-7979(2009)0000020014.

Hoskisson, R., Eden, L., Lau, C. \& Wright, M. (2000). Strategy in emerging economies. Academy of Management Journal, 43(3), 249-267. https://doi.org/10.5465/1556394

Hung, K. \& Chou, C. (2013). The impact of open innovation on firm performance: The moderating effects of internal RyD and environmental turbulence. Technovation, 33(10), 368-380. https://doi.org/10.1016/j.technovation.2013.06.006

Hurmelinna, L. \& Olander, H. (2014). Coping with rivals' absorptive capacity in innovation activities. Technovation, 34(1), 3-11. https://doi.org/10.1016/j.technovation.2013.07.005 
Jansen , J., Van den Bosch, F. \& Volberda, H. (2006). Exploratory innovation, exploitative innovation and performance: Effects of organizational antecedents and environmental moderators. Management Science, 52(11), 1161-1174. https://doi.org/10.1287/ mnsc.1060.0576

Jansen, J., Van den Bosch, F. \& Volberda, H. (2005). Managing potential and realized absorptive capacity: How do organizational antecedents matter? Academy of Management Journal, 48(6), 999-1015. https://doi.org/10.5465/amj.2005.19573106

Jiao, H., Chang, I. C. \& Lu, Y. (2008). The relationship on information technology capability and performance: An empirical research in the context of China's Yangtze River delta region. In 2008 IEEE International Conference on Industrial Engineering and Engineering Management (pp. 872-876). IEEE.

Judge, W. \& Douglas, T. (1998). Performance implications of incorporating natural environmental issues into the strategic planning process: An empirical assessment. Journal of Management Studies, 35(2), 241-262. https://doi.org/10.1111/1467-6486.00092

Lane, P. \& Lubatkin, M. (1998). Relative absorptive capacity and interorganizational learning. Strategic Management Journal, 19(5), 461-477. https://doi.org/10.1002/(SICI)1097-0 266(199805)19:5<461::AID-SMJ953>3.0.C0;2-L

Lane, P., Koka, B. \& Pathak, S. (2006). The reification of absorptive capacity: A critical review and rejuvenation of the construct. Academy of Management Review, 31(4), 833-863. https://doi.org/10.2307/20159255

Lane, P., Salk, J. \& Lyles, M. (2001). Absorptive capacity, learning, and performance in international joint ventures. Strategic Management Journal, 22(12), 1139-1161. https://doi. org/10.1002/smj.206

Laursen, K. \& Salter, A. (2006). Open for innovation: The role of openness in explaining innovation performance among U.K. manufacturing firms. Strategic Management Journal, 27(2), 131-150. https://doi.org/10.1002/smj.507

Lee, Y., Kim, S. \& Lee, H. (2011). The impact of service RyD on the performance of Korean information communication technology small and medium enterprises. Journal of Engineering and Technology Management, 28(1), 77-92. https://doi.org/10.1016/j. jengtecman.2010.12.005

Lichtenthaler, U. (2009). Absorptive capacity, environmental turbulence, and the complementarity of organizational learning processes. Academy of Management Journal, 52(4), 822-846. https://doi.org/10.5465/amj.2009.43670902

Lieberman, M. \& Montgomery, D. (1988). First-mover advantages. Strategic management Journal, 9(S1), 41-58. https://doi.org/10.1002/smj.4250090706 
Lin, B. W. (2007). Information technology capability and value creation: Evidence from the US banking industry. Technology in Society, 29(1), 93-106. https://doi.org/10.1016/j. techsoc.2006.10.003

Liu, H., Ke, W., Wei, K. \& Hua, Z. (2013). The impact of IT capabilities on firm performance: The mediating roles of absorptive capacity and supply chain agility. Decision Support Systems, 54(3), 1452-1462. https://doi.org/10.1016/j.dss.2012.12.016

Liu, Y., Lu, H. \& Hu, J. (2008). IT capability as moderator between IT investment and firm performance. Tsinghua Science y Technology, 13(3), 329-336. https://doi.org/10.1016/ S1007-0214(08)70053-1

Lu, Y. \& Ramamurthy, K. (2011). Understanding the link between information technology capability and organizational agility: An empirical examination. MIS Quarterly, 35(4), 931-954.

MinTIC (2015). Panorama TIC. Bogotá: Ministerio de Tecnologías de la Información y las Comunicaciones.

Mohd Ishan, Z. \& Noordin, N. (2015). Capitalising on income approach as trademark valuation for entrepreneurs. Pertanika Journal of Social Sciences y Humanities, 23, 147-160.

Nakata, C., Zhu, Z. \& Kraimer, M. (2008). The complex contribution of information technology capability to business performance. Journal of Managerial Issues, 20(4), 485-506.

Podsakoff, P., MacKenzie, S., Lee, J. \& Podsakoff, N. (2003). Common method biases in behavioral research: A critical review of the literature and recommended remedies. Journal of applied psychology, 88(5), 879-903. https://doi: 10.1037/0021-9010.88.5.879

Prajogo, D. \& Ahmed, P. (2006). Relationships between innovation stimulus, innovation capacity, and innovation performance. RyD Management, 36(5), 499-515. https://doi. org/10.1111/j.1467-9310.2006.00450.x

Preacher, K. \& Hayes, A. (2008). Asymptotic and resampling strategies for assessing and comparing indirect effects in multiple mediator models. Behavior Research Methods, 40(3), 879-891. doi: 10.3758/BRM.40.3.879

Richard, P., Devinney, T., Yip, G. \& Johnson, G. (2009). Measuring organizational performance: Towards methodological best practice. Journal of Management, 35(3), 718-804. https:// doi: $10.1177 / 0149206308330560$

Roberts, P. \& Amit, R. (2003). The dynamics of innovative activity and competitive advantage: The case of Australian retail banking, 1981 to 1995. Organization Science, 14(2), 107122. https://doi.org/10.1287/orsc.14.2.107.14990

Sáenz, M., Revilla, E. \& Knoppen, D. (2014). Absorptive capacity in buyer-supplier relationships: Empirical evidence of its mediating role. Journal of Supply Chain Management, 50(2), 18-40. https://doi.org/10.1111/jscm.12020

Santhanam, R. \& Hartono, E. (2003). Issues in linking information technology capability to firm performance. MIS Quarterly, 27(1), 125-153. https://doi: 10.2307/30036521 
Schiffman, L. \& Kanuk, L. (2010). Comportamiento del consumidor. Naucalpan de Juárez: Pearson Educación de México, S.A. de C.V.

Selnes, F. \& Sallis, J. (2003). Promoting relationship learning. Journal of Marketing, 67(3), 8095. https://doi.org/10.1509/jmkg.67.3.80.18656

Shin, N. (2001). The impact of information technology on financial performance: The importance of strategic choice. European Journal of Information Systems, 10(4), 227-236.

Shocker, A., Srivastava, R. \& Ruekert, R. (1994). Challenges and opportunities facing brand management: An introductionto the special issue. Journal of Marketing Research, 31(2), 149-158. http://dx.doi.org/10.2307/3152190

Sorescu, A., Chandy, R. \& Prabhu, J. (2007). Why some acquisitions do better than others: Product capital as a driver of long-term stock returns. Journal of Marketing Research, 44(1), 57-72. https://doi.org/10.1509/jmkr.44.1.57

Srinivasan, S., Pauwels, K., Silva-Risso, J. \& Hanssens, D. (2009). Product innovations, advertising, and stock returns. Journal of Marketing, 73(1), 24-43. https://doi.org/10.1509/ jmkg.73.1.24

Szulanski, G. (1996). Exploring internal stickiness: Impediments to the transfer of best practice within the firm. Strategic Management Journal, 17(S2), 27-43. https://doi.org/10.1002/ smj.4250171105

Tan, M. \& Teo, T. S. (2000). Factors influencing the adoption of internet banking. Journal of the Association for Information Systems, 1(5), 1-42.

Tanriverdi, H. (2005). Information technology relatedness, knowledge management capability, and performance of multibusiness firms. MIS Quarterly, 29(2), 311-334. https://doi: $10.2307 / 25148681$

Tippins, M. \& Sohi, R. (2003). IT competency and firm performance: Is organizational learning a missing link. Strategic Management Journal, 24(8), 745-761. https://doi.org/10.1002/ smj. 337

Tiwana, A. \& Mclean, E. (2005). Expertise integration and creativity in information systems development. Journal of Management Information Systems, 22(1), 13-43. https://doi> 10.1080/07421222.2003.11045836

Todorova, G. \& Durisin, B. (2007). Absorptive capacity: Valuing a reconceptualization. Academy of Management Review, 32(3), 774-786. https://doi: 10.2307/20159334

UIT (2015). Informe sobre la medición de la sociedad de la información. Ginebra.

Volberda, H., Foss, N. \& Lyles, M. (2010). Absorbing the concept of absorptive capacity: How to realize its potential in the organization field. Organization Science, 21(4), 931-951. http://dx.doi.org/10.2139/ssrn.1513184 
Wales, W., Parida, V. \& Pankaj, C. (2013). Too much of a good thing? absorptive capacity, firm performance, and the moderating role of entrepreneurial orientation. Strategic Management Journal, 34(5), 622-633. https://doi.org/10.1002/smj.2026

Williams, J. \& MacKinnon, D. (2008). Resampling and distribution of the product methods for testing indirect effects in complex models. Structural Equation Modeling, 15(1), 23-51. https://doi.org/10.1080/10705510701758166

WEF (2015). The Global Information Technology Report 2015. Geneva.

Yu, C. \& Xin-quan, G. (2011). The empirical study on the relationship between information technology capability and innovation performance: The moderating role of learning commitment. In E-Business and E-Government (ICEE), International Conference on (pp. 1-4). IEEE.

Zahra, S. \& George, G. (2002). Absorptive capacity: A review, reconceptualization, and extension. Academy of Management Review, 27(2), 185-203. https://doi: 10.2307/4134351

Zawislak, P., Alves, A., Tello-Gamarra, J., Barbieux, D. \& Reichert, F. (2012). Innovation capability: from technology development to transaction capability. Journal of Technology Management and Innovation, 7(2), 14-27. doi: 10.4067/S0718-27242012000200002

Zhang, M. \& Tansuhaj, P. (2007). Organizational culture, information technology capability, and performance: The case of born global firms. Multinational Business Review, 15(3), 43-78. https://doi.org/10.1108/1525383X200700012

\section{Anexo}

\begin{tabular}{|c|c|}
\hline CTI1 & $\begin{array}{l}\text { Los servicios de TI para almacenar, compartir y facilitar el acceso a datos e información (por } \\
\text { ejemplo, bases de datos, servicios en la nube), son: }\end{array}$ \\
\hline CTI2 & $\begin{array}{l}\text { Los servicios de TI para las comunicaciones en línea (por ejemplo, videoconferencias, red } \\
\text { social corporativa), son: }\end{array}$ \\
\hline СТI3 & $\begin{array}{l}\text { El software y las aplicaciones que apoyan la operación del negocio (por ejemplo, ERP, CRM), } \\
\text { son: }\end{array}$ \\
\hline CTI4 & $\begin{array}{l}\text { El hardware y las instalaciones físicas para la prestación de servicios de TI (por ejemplo, } \\
\text { datacenter, servidores), son: }\end{array}$ \\
\hline CTI5 & El desarrollo de una visión clara respecto a cómo las TI agregan valor al negocio, ha sido: \\
\hline CTI6 & La alineación de la planeación estratégica del negocio con la estrategia de TI, ha sido: \\
\hline CTI7 & $\begin{array}{l}\text { El desarrollo del área de TI y de las capacidades gerenciales para entender el valor de las } \\
\text { inversiones en TI, ha sido: }\end{array}$ \\
\hline CTI 8 & $\begin{array}{l}\text { La empresa se mantiene actualizada con la información más reciente sobre innovaciones } \\
\text { tecnológicas. }\end{array}$ \\
\hline CTI 9 & $\begin{array}{l}\text { La empresa tiene la capacidad de experimentar con nuevas Tecnologías de Información (TI) } \\
\text { cuando es necesario. }\end{array}$ \\
\hline
\end{tabular}


CTI: Capacidades de las Tecnologías de la Información (Lu y Ramamurthy, 2011)

\begin{tabular}{|c|c|}
\hline \multicolumn{2}{|r|}{ CTI: Capacidades de las Tecnologías de la Información (Lu y Ramamurthy, 2011) } \\
\hline CTI 10 & $\begin{array}{l}\text { En la empresa hay un clima que apoya y aprueba nuevas formas de utilizar las Tecnologías de } \\
\text { Información (TI). }\end{array}$ \\
\hline CTI 11 & $\begin{array}{l}\text { Se buscan constantemente nuevas formas de mejorar la eficacia en el uso de Tecnologías de } \\
\text { Información (TI). }\end{array}$ \\
\hline \multicolumn{2}{|r|}{ DF: Desempeño Financiero (Judge y Douglas, 1998) } \\
\hline DF1 & El retorno sobre la inversión \\
\hline DF2 & El crecimiento de las ganancias \\
\hline DF3 & El crecimiento en ventas \\
\hline DF4 & El aumento de la participación de mercado \\
\hline \multicolumn{2}{|r|}{ DnF: Desempeño No Financiero (Lee et al., 2011) } \\
\hline DnF1 & El aumento en la satisfacción del cliente \\
\hline DnF2 & El mejoramiento de la imagen corporativa \\
\hline DnF3 & El aumento del valor de las marcas \\
\hline DnF4 & El aumento de la productividad de los empleados \\
\hline \multicolumn{2}{|r|}{ CAB: Capacidad de Absorción (Flatten, Engelena, Zahra y Brettel, 2011) } \\
\hline CAB1 & $\begin{array}{l}\text { La búsqueda de información relevante sobre la industria a la que pertenece la empresa es una } \\
\text { tarea de todos los días. }\end{array}$ \\
\hline САВ2 & $\begin{array}{l}\text { Los directivos motivan a los empleados a utilizar fuentes de información sobre el sector al que } \\
\text { pertenece la empresa. }\end{array}$ \\
\hline CAB3 & $\begin{array}{l}\text { Los directivos esperan que los empleados estén familiarizados con información de otras } \\
\text { industrias distintas a la que pertenece la empresa. }\end{array}$ \\
\hline CAB4 & Se intercambian ideas y conceptos entre las distintas áreas funcionales de la empresa. \\
\hline CAB5 & Los directivos fomentan el apoyo entre las distintas áreas de la empresa para resolver problemas. \\
\hline CAB6 & $\begin{array}{l}\text { La información fluye rápidamente entre los colaboradores, por ejemplo, si un área adquiere } \\
\text { información importante, la comunica rápidamente a las otras áreas de la empresa. }\end{array}$ \\
\hline CAB7 & $\begin{array}{l}\text { Los directivos promueven reuniones periódicas entre áreas funcionales para intercambiar } \\
\text { nuevos desarrollos, problemas y logros. }\end{array}$ \\
\hline CAB8 & $\begin{array}{l}\text { Los empleados tienen la capacidad de estructurar y utilizar el conocimiento externo que ha } \\
\text { adquirido la empresa. }\end{array}$ \\
\hline CAB9 & $\begin{array}{l}\text { Los empleados usualmente absorben nuevo conocimiento y lo organizan para que esté } \\
\text { disponible para otros y pueda ser usado posteriormente }\end{array}$ \\
\hline CAB10 & $\begin{array}{l}\text { Los empleados articulan de manera exitosa los conocimientos existentes con nuevas ideas y } \\
\text { puntos de vista. }\end{array}$ \\
\hline CAB11 & Los empleados son capaces de aplicar los nuevos conocimientos en su trabajo. \\
\hline CAB12 & Los directivos apoyan el desarrollo de prototipos. \\
\hline CAB13 & La empresa regularmente reevalúa tecnologías existentes y las adapta de manera novedosa. \\
\hline CAB14 & $\begin{array}{l}\text { La empresa tiene la capacidad de trabajar de manera más efectiva mediante la adopción de } \\
\text { nuevas tecnologías. }\end{array}$ \\
\hline
\end{tabular}

